\title{
Development of a Patient-Reported Outcome Measure for Chronic Hypoparathyroidism
}

\author{
Susan Martin · Kristina Chen · Nimanee Harris • Montserrat Vera-Llonch • \\ Alan Krasner
}

Received: February 22, 2019 / Published online: June 10, 2019

(C) The Author(s) 2019

\section{ABSTRACT}

Introduction: A patient-reported outcome (PRO) measure specific to chronic hypoparathyroidism is lacking to facilitate the evaluation of treatment. A PRO measure that followed the recommendations of the US Food and Drug Administration (FDA) PRO guidance was created to address key hypoparathyroidism symptoms.

Methods: A literature review was conducted to identify symptoms of hypoparathyroidism and any existing PRO measures appropriate for

Enhanced Digital Features To view enhanced digital features for this article go to https://doi.org/10.6084/ m9.figshare.8138234.

S. Martin $(\bowtie)$

RTI Health Solutions, Ann Arbor, MI, USA

e-mail: smartin@rti.org

K. Chen · M. Vera-Llonch

Shire Human Genetic Therapies, Inc., A Member of the Takeda Group of Companies, Cambridge, MA, USA

N. Harris

RTI Health Solutions, Research Triangle Park, NC, USA

\section{A. Krasner}

Shire Human Genetic Therapies, Inc., A Member of the Takeda Group of Companies, Lexington, MA, USA

Present Address:

A. Krasner

Crinetics Pharmaceuticals, San Diego, CA, USA evaluating these symptoms, followed by concept elicitation interviews involving six individuals with hypoparathyroidism. On the basis of the results of the literature review and interviews, a draft item pool was developed and refined through two sets of cognitive debriefing interviews with six additional patients. A translatability assessment was also conducted to evaluate concepts, phrases, or components of the items that could be problematic in future translations and to identify culturally specific phrasing.

Results: No PRO measures appropriate to address hypoparathyroidism symptoms documented in the literature were identified. Qualitative research participants included 11 women and one man, with an average age of 49 years; the majority (10) of these participants were white. Concept elicitation interview results were generally consistent with the results of the literature review; the most commonly reported symptoms included issues with cognition, often described as "brain fog" $(n=6)$, muscle cramping $(n=5)$, tingling $(n=5)$, and muscle spasms or twitching $(n=4)$. The initial draft item pool included 20 items; based upon participant feedback, the final Hypoparathyroidism Symptom Diary comprised 13 items and was found to be easily understood and relevant to the participants. No significant issues were identified by the translatability assessment.

Conclusion: The Hypoparathyroidism Symptom Diary was developed following the 
recommendations of the FDA's PRO guidance. The measure addresses a comprehensive set of symptoms, as well as key impacts of hypoparathyroidism deemed important by patients.

Funding: Shire Human Genetic Therapies, Inc., Lexington, MA, USA, a member of the Takeda group of companies.

Keywords: Endocrinology; Hypoparathyroidism; Patient-reported outcomes; Qualitative research; Symptoms

\section{INTRODUCTION}

Hypoparathyroidism is a rare disorder that causes lower than normal levels of calcium in the blood due to insufficient levels of parathyroid hormone [1]. Chronic hypoparathyroidism is most often the result of neck surgeries, but can also be inherited or associated with other disorders [2]. Hypocalcemia due to hypoparathyroidism can affect tissue and organ systems, including the brain, heart, and kidneys, with effects resulting in signs and symptoms experienced by patients [2]. Currently, no patient-reported outcome (PRO) measure specific to the experience of hypoparathyroidism exists. Therefore, the aim of this research was to develop a novel measure to assess symptoms in this patient population that can facilitate the evaluation of new and existing treatments for the condition by incorporating patient experiences in accordance with the United States (US) Food and Drug Administration's (FDA) guidance regarding the use of PRO measures to support labeling claims [3].

\section{METHODS}

This study conformed with the Declaration of Helsinki of 1964, as revised in 2013, concerning human and animal rights, and Springer's policy concerning informed consent has been followed. The study was reviewed and approved by RTI International's institutional review board (IRB), and informed consent was obtained from all individual participants included in the study. Patientidentifying data from the qualitative research was not included in any transcripts or reporting to protect confidentiality. Figure 1 displays a schematic of the instrument development process used in the development of the Hypoparathyroidism Symptom Diary, and the methods associated with each phase of the development are detailed below. The Hypoparathyroidism Symptom Diary is currently completing validation in accordance with FDA guidance [3].

\section{Concept Identification: Literature Review and Concept Elicitation Interviews}

Potential symptom concepts for the new measure were identified through a review of the literature. Specifically, the authors (SM and $\mathrm{NH}$ ) conducted a PubMed search focused on studies of chronic hypoparathyroidism (search terms included "chronic hypoparathyroidism," "chronic HypoPT, Hypoparathyroidism" [MeSH] and "Chronic Disease" [MeSH]) published from 2005 through 2015, resulting in 30 identified abstracts. Of these, seven abstracts were selected for full-text review as the titles and/or abstract text indicated that the publication included symptoms of hypoparathyroidism; 23 were excluded for no indication that the publication included symptoms related to hypoparathyroidism or because the article focused on another disease or condition. Upon completion of the full-text review, four articles were included in the review based on inclusion of key symptoms of chronic hypoparathyroidism. The three excluded articles described research pertaining to cellular immunity, animal models, genetic patterns, and clinician market research. In addition to these articles identified by the search, one of the authors (KC) provided three articles that included relevant data regarding symptoms, bringing the total number of reviewed publications to seven. In addition, a desktop search of unpublished ("gray") literature sources, including all meeting abstracts from the 2015 American Society for Bone and Mineral Research conference and an Internet search of materials developed for patient education, was conducted and identified a fact sheet produced by the Endocrine Society, which was included in the review of symptoms (no relevant abstracts were identified from the conference proceedings). 


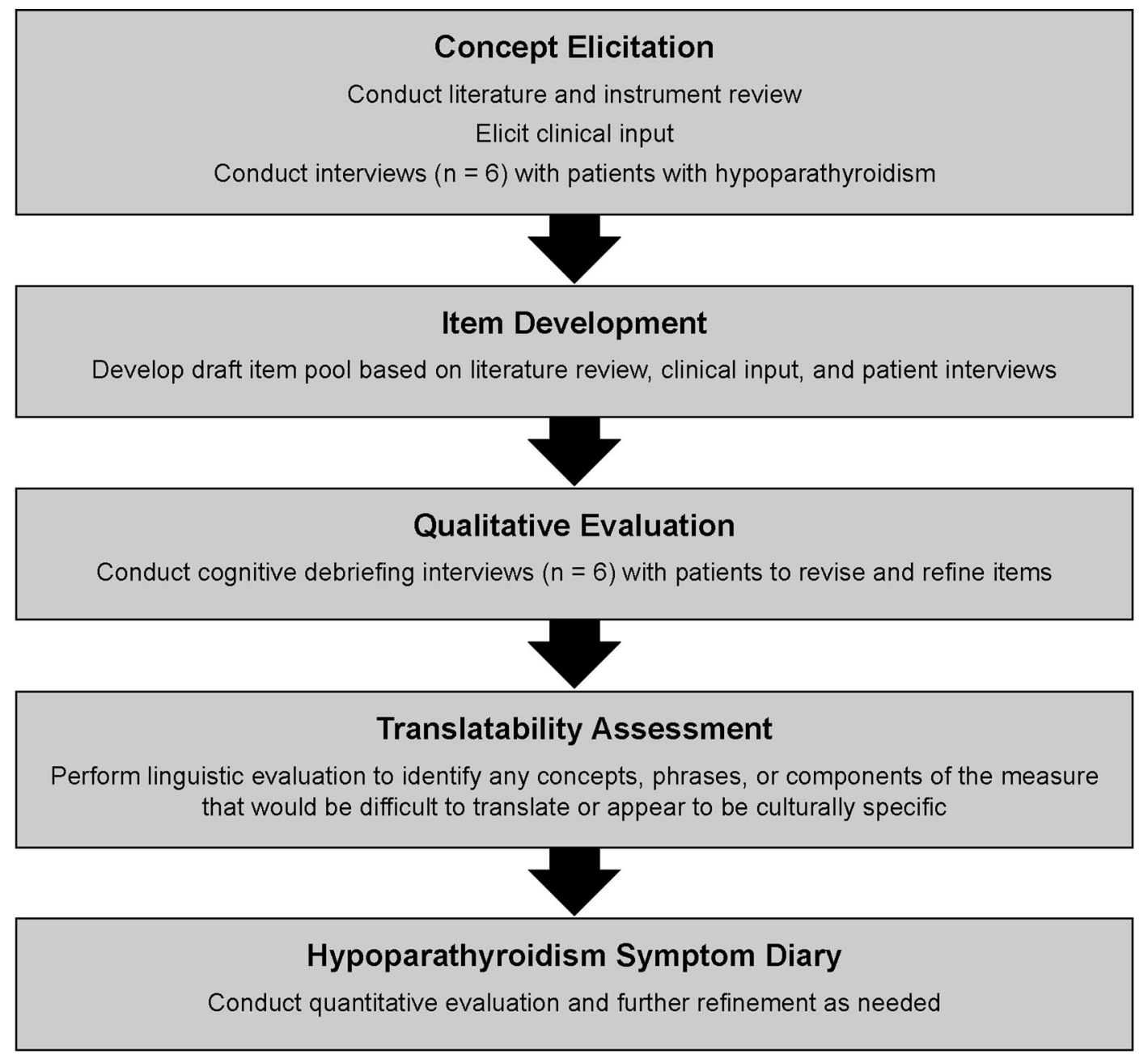

Fig. 1 Hypoparathyroidism Symptom Diary development process

Upon completion of the literature review, concept elicitation interviews were conducted via telephone with six participants with hypoparathyroidism residing in the USA. Recruitment was conducted in collaboration with the Hypoparathyroidism Association, a patient advocacy group, that shared IRB-approved recruitment text about the research study with individuals from the association's database to identify potential participants that were interested and met the study criteria. Eligible participants met the following self-reported inclusion criteria: adult (aged at least 18 years) with a physician diagnosis of chronic hypoparathyroidism; currently taking oral calcium and active vitamin D for the treatment of hypoparathyroidism; experiencing symptoms due to chronic hypoparathyroidism despite ongoing treatment; willing and able to participate in a 1-h interview; able to read, speak, and understand English; and willing and able to provide informed consent for participation in a research study. Participants were excluded from the concept elicitation interviews if they had the CASR gene mutation or an impaired responsiveness to parathyroid hormone or if they had been or were currently treated with recombinant human parathyroid hormone (1-84). Interviews were conducted using an interview guide, which allowed for open-ended discussion and spontaneous reports of hypoparathyroidism symptoms and also included probe questions (after the 
open-ended portion), that was based on the results of the literature review.

\section{Item Development}

On the basis of the results of the targeted literature review and the concept elicitation interviews, a draft item pool using standard survey methodology was developed. Specifically, the items were developed using the terminology used by participants, and alternative item wording and response options (i.e., numerical rating scales and verbal rating scales) were developed for further evaluation with the participants. The draft item pool was also reviewed by an endocrinologist (AK) prior to finalizing the items for the cognitive debriefing interviews.

\section{Qualitative Evaluation}

To pretest and refine the draft item pool, two iterative rounds of in-person cognitive debriefing interviews were conducted with six additional participants identified with the assistance of the Hypoparathyroidism Association. Participants met the same criteria as detailed for the concept elicitation interviews (described earlier). As with the concept elicitation interviews, the cognitive debriefing interviews followed a semistructured interview guide. Specifically, participants were asked to respond and provide feedback on the draft item pool using a "thinkaloud" process [4] to ensure that the final question wording and response options were easily understood and interpreted consistently. The interviewers also asked whether important symptoms or concepts were missing.

\section{Translatability Assessment}

After conducting the first three cognitive interviews, a translatability assessment was conducted to identify any concepts, phrases, or components of the instrument that would be difficult to translate or that appeared to be culturally specific. A team of eight linguists and native speakers of the following language groups reviewed the measure in development: English (UK), Spanish (Spain), French (France),
German (Germany), Italian (Italy), Norwegian (Norway), Danish (Denmark), and Swedish (Sweden). The results of the translatability assessment then were reviewed by a survey research expert and a quality assurance project manager to discuss any changes that should be made to the source text based on the results.

\section{RESULTS}

\section{Concept Identification: Literature Review and Concept Elicitation Interviews}

No hypoparathyroidism-specific PRO measures were identified in the literature or desktop review. Of the seven reviewed studies, three were case studies [5-7], one was a review article [8], one was the proceedings of an expert panel [9], one was a clinical trial [10], and one was an observational study [11]. The observational study provided the greatest level of detail regarding symptoms reported by patients with hypoparathyroidism and was based on a survey completed by 374 adults with the condition.

Table 1 provides a summary of the symptoms identified from the review. The most commonly cited hypoparathyroidism symptoms identified across the articles were muscle cramps in the limbs, feet, fingers, stomach, or face. Other symptoms commonly cited were muscle spasms; tingling in the fingers, toes, and lips; weakness; muscle pain or discomfort; numbness or pain in the fingers, toes, and lips; and memory problems. One of the reviewed articles [11] described the most frequent symptoms (those reported via a symptom checklist by more than $50 \%$ of 374 patients with hypoparathyroidism) as fatigue; muscle pain or cramping; paresthesia; brain fog or mental lethargy; tetany; joint or bone pain; inability to focus or concentrate; memory loss or forgetfulness; anxiety or fear or inner unrest; sleep disturbances; pain, heaviness, or weakness in extremities; and feeling sad, down, blue, or depressed.

The literature review results were incorporated into the next phase of the research, the concept elicitation interviews. Specifically, these results were used to create a list of symptom probes for follow-up with the participants 
Table 1 Hypoparathyroidism symptoms reported in reviewed sources

\begin{tabular}{ll}
\hline Symptom & References \\
\hline Muscle cramps in the limbs, feet, fingers, stomach, or face & {$[7-11,15]$} \\
Muscle spasms in the limbs, feet, fingers, stomach, or face & {$[6-9,15]$} \\
Paresthesia (tingling) in the fingers, toes, and lips & {$[7,9,11,15]$} \\
Weakness & {$[7,9,11,15]$} \\
Muscle pain or discomfort in the limbs, feet, fingers, stomach, or face & {$[7-9,15]$} \\
Numbness of the fingers or limbs & {$[6,11]$} \\
Pain of the fingers or limbs & {$[11]$} \\
Memory problems including "brain fog" or memory loss & {$[7,9,11,15]$} \\
Depression or feeling depressed & {$[5,11,15]$} \\
Chest pain & {$[2,8,9]$} \\
Respiratory problems, bronchospasm, or laryngospasm & {$[7,9,11]$} \\
Chronic headaches & {$[11]$} \\
Anxiety & {$[5,11]$} \\
Pain with menstrual periods & {$[15]$} \\
Heaviness in extremities & {$[11]$} \\
Joint or bone pain & {$[11]$} \\
Disturbance to bowel movements & {$[11]$} \\
Fatigue & {$[11]$} \\
Sleep disturbances & {$[11]$} \\
Gait impacts (shuffling or frozen) ${ }^{a}$ & {$[6]$} \\
\hline
\end{tabular}

a One case study patient

upon conclusion of the open-ended concept elicitation portion of the interview.

Participant characteristics are provided in Table 2. The mean age across participants $(n=6)$ was 53 years (range 32-71), and the mean number of years with hypoparathyroidism across the participants was 17 (range $7-44)$. Most were female $(n=5)$ and white $(n=5)$. All had at least some college education, with half being employed full-time and half being unemployed or retired.

Table 3 provides the spontaneous and probed symptoms reported by two or more participants in the six concept elicitation interviews. The results were generally consistent with those of the literature review. All participants spontaneously reported issues with cognition, and all but one also spontaneously reported the symptoms muscle cramps and tingling or numbness. All but two participants spontaneously reported muscle spasms or twitching. Fatigue was also reported by all participants, either spontaneously $(n=3)$ or upon probing $(n=3)$.

\section{Item Development}

On the basis of the results from the literature review, clinical input, and concept elicitation 
Table 2 Participant characteristics: concept elicitation and cognitive debriefing

\begin{tabular}{llll}
\hline Characteristic & $\begin{array}{l}\text { Concept elicitation } \\
\text { interviews }(\boldsymbol{n}=\mathbf{6})\end{array}$ & $\begin{array}{l}\text { Cognitive debriefing } \\
\text { interviews }(\boldsymbol{n}=\mathbf{6})\end{array}$ & Total $(\boldsymbol{n}=\mathbf{1 2})$ \\
\hline Sex, female & $5(83)$ & $6(100)$ & $11(92)$ \\
Age in years, mean (range) & $53(32-71)$ & $44(33-69)$ & $49(32-71)$ \\
Years with hypoparathyroidism, mean (range) & $17(7-44)$ & $15(2-40)$ & $16(2-44)$ \\
Race/ethnicity & & $5(83)$ & $10(83)$ \\
White & $5(83)$ & - & $1(8)$ \\
Asian & $1(17)$ & $1(17)$ & $1(8)$ \\
Native American & - & $3(50)$ & $5(42)$ \\
Education & & $3(50)$ & $5(42)$ \\
Some college & $2(33)$ & - & $2(17)$ \\
College degree & $2(33)$ & & $7(58)$ \\
Professional/advanced degree & $2(33)$ & $4(67)$ & $1(8)$ \\
Employment & & $1(17)$ & $4(33)$ \\
Full-time & $3(50)$ & $1(17)$ & \\
Part-time & - & $3(50)$ & \\
Unemployed/retired &
\end{tabular}

Data are presented as $n(\%)$

interviews, an initial item pool was developed. The item pool included 20 items assessing the severity of 10 symptoms (two item options were developed for each symptom). The symptoms assessed were muscle cramps, tingling or numbness, muscle spasms or twitching, muscle pain or soreness, bone or joint pain, feelings of body weakness or heaviness, tiredness or fatigue, slowed or confused thinking, feelings of fear or anxiety, and feeling sad or depressed. Alternate question wording and response options, including verbal rating scales and numerical rating scales (ranging from 0 to 10), were developed for each of the symptom items. In addition, on the basis of the results of the interviews and the clinical input (AK), three areas of impact (nonsymptom items) were identified for further evaluation with patients with hypoparathyroidism: the impact of hypoparathyroidism on sleep, ability to exercise, and work.

\section{Qualitative Evaluation (Cognitive Debriefing Interviews)}

Participant characteristics are provided in Table 2. The mean age across participants $(n=6)$ was 44 years (range 33-69), and the mean number of years with hypoparathyroidism across the participants was 15 years (range 2-40). All were female $(n=6)$, and most were white $(n=5)$. All had at least some college education, and most were working either fulltime $(n=4)$ or part-time $(n=1)$.

On the basis of the feedback from the first three cognitive debriefing participants, the draft item pool was revised to include two additional symptom items. Specifically, participants identified that two of the original symptoms needed to be separate symptom concepts. Therefore, "numbness and tingling" became two separate items, and similarly, "bone and joint pain" became two separate items. No modifications were made to the impact items. 
Table 3 Hypoparathyroidism symptom summary table: concept elicitation interviews

\begin{tabular}{|c|c|c|c|c|c|c|c|}
\hline $\begin{array}{l}\text { Symptom (participant } \\
\text { terms for symptom) }\end{array}$ & $\begin{array}{l}\text { Participant } \\
1\end{array}$ & $\begin{array}{l}\text { Participant } \\
2\end{array}$ & $\begin{array}{l}\text { Participant } \\
3\end{array}$ & $\begin{array}{l}\text { Participant } \\
4\end{array}$ & $\begin{array}{l}\text { Participant } \\
5\end{array}$ & $\begin{array}{l}\text { Participant } \\
6\end{array}$ & Tota \\
\hline $\begin{array}{l}\text { Cognitive issues (brain } \\
\text { fog, confused thinking, } \\
\text { loss of concentration, } \\
\text { short-term memory) }\end{array}$ & $S$ & $S$ & $S$ & $S$ & $S$ & $S$ & S: 6 \\
\hline Muscle cramping & $S$ & & $S$ & $S$ & $S$ & $S$ & $S: 5$ \\
\hline Tingling or numbness & $S$ & & $S$ & $S$ & $S$ & $S$ & $S: 5$ \\
\hline $\begin{array}{l}\text { Muscle spasms } \\
\text { (twitching) }\end{array}$ & $S$ & $S$ & $S$ & & $S$ & & S: 4 \\
\hline $\begin{array}{l}\text { Fatigue (fatigue, } \\
\text { tiredness, exhaustion) }\end{array}$ & $S$ & $S$ & $S$ & $\mathrm{P}$ & $\mathrm{P}$ & $S$ & $\begin{array}{l}\text { S: } 4 \\
\text { P: } 2\end{array}$ \\
\hline Feeling anxious or fearful & $S$ & & $\mathrm{P}$ & $S$ & $S$ & $\mathrm{P}$ & $\begin{array}{l}\text { S: } 3 \\
\text { P: } 2\end{array}$ \\
\hline Joint or bone pain & $S$ & $S$ & $\mathrm{P}$ & & $\mathrm{P}$ & $\mathrm{P}$ & $\begin{array}{l}\text { S: } 2 \\
\text { P: } 3\end{array}$ \\
\hline $\begin{array}{l}\text { Muscle soreness and } \\
\text { muscle pain }\end{array}$ & $\mathrm{P}$ & $S$ & & & $S$ & & $\begin{array}{l}S: 2 \\
P: 1\end{array}$ \\
\hline $\begin{array}{l}\text { Feeling depressed } \\
\text { (emotional numbness, } \\
\text { tearful, hypersensitive) }\end{array}$ & & & & $\mathrm{P}$ & $S$ & $S$ & $\begin{array}{l}S: 2 \\
P: 1\end{array}$ \\
\hline $\begin{array}{l}\text { Pain, heaviness, or } \\
\text { weakness in your arms } \\
\text { or legs }\end{array}$ & & $\mathrm{P}$ & $\mathrm{P}$ & & $\mathrm{P}$ & $S$ & $\begin{array}{l}\text { S: } 1 \\
P: 3\end{array}$ \\
\hline $\begin{array}{l}\text { Trouble breathing or } \\
\text { feeling like your throat } \\
\text { is closing }\end{array}$ & & & & $S$ & $S$ & & $S: 2$ \\
\hline Headache & & & $S$ & & & $S$ & $S: 2$ \\
\hline Constipation & & $S$ & & & S & & $S: 2$ \\
\hline $\begin{array}{l}\text { Dry mouth, feeling } \\
\text { thirsty, or dehydration }\end{array}$ & & & $S$ & & $S$ & & $S: 2$ \\
\hline $\begin{array}{l}\text { Ears ringing or loud } \\
\text { shushing sound }\end{array}$ & & & & $S$ & $S$ & & S: 2 \\
\hline
\end{tabular}

$P$ reported upon probing, $S$ reported spontaneously

Upon completion of the next three cognitive debriefing interviews, three symptom items were omitted from the final Hypoparathyroidism
Symptom Diary, and one impact item was added. Specifically, participants reported that muscle pain or soreness, bone pain, and joint pain may 
be associated with other issues rather than their hypoparathyroidism. In addition, an impact item was included to assess family relationships on the basis of the feedback provided about missing concepts described below.

When asked to identify any missing concepts, only two of the six participants described a missing symptom concept, and four participants described a missing impact concept. Regarding symptoms, one participant stated that an inability to regulate body temperature was a missing symptom, while another participant described slowed speech. Inability to regulate body temperature had not been described in the literature or by any of the previous concept elicitation or cognitive debriefing participants, so this symptom was not included. On the basis of evaluation of the participant responses to the item regarding slowed or confused thinking (brain fog), it appeared that the issue regarding slowed speech was being captured in the existing item, so this was also not added as a new item. Regarding the impact items, one participant mentioned including a question about how hypoparathyroidism psychologically impacts lives given that the condition does not have any visible symptoms and therefore others may not perceive it as a serious condition or provide support when needed. Another participant discussed the impact on quality of life, as well as impacts on mood, such as irritability. Finally, two participants mentioned that the impact on family relationships was missing from the questionnaire. For the impacts mentioned by only one participant, given that the primary focus of the diary is to assess symptoms and include key impacts, the two impacts mentioned by only one participant each were not included; however, as two participants reported family relationships as missing, this item was added to the final measure.

The concepts included in the final Hypoparathyroidism Symptom Diary (Table 4) were deemed relevant, were easy to understand and answer, and were interpreted consistently by the interview participants. Participants did not report difficulty recalling the symptoms and impacts experienced over the past $24 \mathrm{~h}$ (the stated recall period).

\section{Translatability Assessment}

No significant issues related to translation of the measure or cultural specificity were noted.

\section{DISCUSSION}

Recent clinical guidelines have highlighted the importance of the symptoms experienced by patients with hypoparathyroidism in determining the appropriate treatment approach [12] and have identified preventing signs and symptoms as a key goal of chronic management therapy [1]. However, no standardized assessment of symptoms in this rare condition has been available until now. Development of the Hypoparathyroidism Symptom Diary will allow patient report of the symptoms and key impacts of the condition, which can be used in future clinical trials and, given the brevity of the measure, may also have utility in clinical practice.

One limitation to this research is the relatively small sample size (a total of 12 participants). However, the sample size was determined in the context of hypoparathyroidism as a rare disease, and, as recommended by recently published recommendations regarding clinical outcome assessment in rare diseases [13], the qualitative results were supplemented by the results of the literature review, as well as by clinical review and input. Importantly, though this sample size was small, the results were consistent across the participant interviews with regard to the symptoms experienced by patients with hypoparathyroidism, indicating that the sample size was sufficient for the development of the new symptom diary.

Another significant limitation of the research was that-consistent with the recognized higher prevalence of hypoparathyroidism in women-only one man participated in the study. While the symptoms and symptom descriptions provided by the male participant were consistent with those described by the female participants, recruitment of additional male participants would have been preferable and should be a goal of further research. 
Table 4 Hypoparathyroidism Symptom Diary final item concepts

\begin{tabular}{|c|c|}
\hline Item concept & Response scale \\
\hline \multicolumn{2}{|l|}{ Symptoms } \\
\hline Muscle cramps & VRS (5 levels: none to very severe $)^{a}$ \\
\hline Tingling & VRS (5 levels: none to very severe) \\
\hline Numbness & VRS (5 levels: none to very severe) \\
\hline Muscle spasms & VRS (5 levels: none to very severe) \\
\hline Heaviness in arms or legs & VRS (5 levels: none to very severe) \\
\hline Physical fatigue & VRS (5 levels: none to very severe) \\
\hline Slowed or confused thinking (brain fog) & VRS (5 levels: none to very severe) \\
\hline Anxiety & VRS ( 5 levels: none to very severe) \\
\hline Sadness or depression & VRS (5 levels: none to very severe) \\
\hline \multicolumn{2}{|l|}{ Impacts } \\
\hline Sleep & VRS (3 levels: not at all to very much) \\
\hline Ability to exercise & VRS (3 levels: not at all to very much) \\
\hline Ability to work (at school, home, or at a job) & VRS (3 levels: not at all to very much) \\
\hline Relationships with family members & VRS (3 levels: not at all to very much) \\
\hline
\end{tabular}

Finally, this research comprised a sample of patients associated with a patient advocacy organization that reported experiencing symptoms despite ongoing treatment for chronic hypoparathyroidism and therefore may not be representative of all patients with chronic hypoparathyroidism.

\section{CONCLUSION}

The Hypoparathyroidism Symptom Diary was developed in accordance with the FDA's PRO guidance [3]. The measure addresses a comprehensive set of symptoms, as well as key impacts of hypoparathyroidism deemed important to patients. The resulting data can provide an assessment of the patient experience, in particular symptoms, in order to fully characterize treatment impacts. The measure is relatively brief and would be reasonable to administer on a daily basis in the context of clinical studies. Further research is under way to develop the scoring algorithm and evaluate the measure's psychometric properties in the context of observational studies in this patient population [14].

\section{ACKNOWLEDGEMENTS}

Funding. Sponsorship for this study, the article processing charges, and the open access fee were funded by Shire Human Genetic Therapies, Inc., Lexington, MA, USA, a member of the Takeda group of companies. All authors had full access to all of the data in this study 
and take complete responsibility for the integrity of the data and accuracy of the data analysis.

Editorial Assistance. Editorial assistance in the preparation of this article was provided by Adele Monroe of RTI Health Solutions (Research Triangle Park, North Carolina, USA), and was funded by Shire Human Genetic Therapies, Inc., a member of the Takeda group of companies.

Authorship. All named authors meet the International Committee of Medical Journal Editors (ICMJE) criteria for authorship for this article, take responsibility for the integrity of the work as a whole, and have given their approval for this version to be published.

Disclosures. Susan Martin is an employee of RTI Health Solutions, a unit of RTI International, a not-for-profit research organization that provides consulting and research services to governmental organizations, businesses, and pharmaceutical companies. Nimanee Harris is an employee of RTI Health Solutions, a unit of RTI International, a not-for-profit research organization that provides consulting and research services to governmental organizations, businesses, and pharmaceutical companies. Kristina Chen is an employee of Shire Human Genetic Therapies, Inc., a member of the Takeda group of companies. Montserrat Vera-Llonch is an employee of Shire Human Genetic Therapies, Inc., a member of the Takeda group of companies. Alan Krasner is a former employee of Shire Human Genetic Therapies, Inc, a member of the Takeda group of companies, and is currently affiliated with Crinetics Pharmaceuticals, San Diego, CA, USA.

Compliance with Ethics Guidelines. This study conformed with the Declaration of Helsinki of 1964, as revised in 2013, concerning human and animal rights, and Springer's policy concerning informed consent has been followed. The study was reviewed and approved by RTI International's institutional review board. All procedures performed in studies involving human participants were in accordance with the ethical standards of the institutional and/or national research committee and with the 1964 Helsinki declaration and its later amendments or comparable ethical standards. Informed consent was obtained from all individual participants included in the study.

Data Availability. Data sharing is not applicable to this article as no data sets were generated or analyzed during the current study.

Open Access. This article is distributed under the terms of the Creative Commons Attribution-NonCommercial 4.0 International License (http://creativecommons.org/licenses/ by-nc/4.0/), which permits any noncommercial use, distribution, and reproduction in any medium, provided you give appropriate credit to the original author(s) and the source, provide a link to the Creative Commons license, and indicate if changes were made.

\section{REFERENCES}

1. Brandi ML, Bilezikian JP, Shoback D, et al. Management of hypoparathyroidism: summary statement and guidelines. J Clin Endocrinol Metab. 2016;101:2273-83.

2. Shoback DM, Bilezikian JP, Costa AG, et al. Presentation of hypoparathyroidism: etiologies and clinical features. J Clin Endocrinol Metab. 2016;101:2300-12.

3. US Department of Health and Human Services, Center for Drug Evaluation and Research (CDER), Center for Biologics Evaluation and Research (CBER), Center for Devices and Radiological Health (CDRH). Guidance for industry patient-reported outcome measures: use in medical product development to support labeling claims. Rockville, MD: US Food and Drug Administration. December 2009. http://www.fda.gov/downloads/Drugs/GuidanceCo mplianceRegulatoryInformation/Guidances/UCM1 93282.pdf. Accessed 30 May 2019.

4. Beatty PC, Willis GB. Research synthesis: the practice of cognitive interviewing. Public Opin Q. 2007;71:287-311.

5. Bohrer T, Krannich JH. Depression as a manifestation of latent chronic hypoparathyroidism. World J Biol Psychiatry. 2007;8:56-9. 
6. Kurozumi A, Okada Y, Arao T, Endou I, Matsumoto T, Tanaka Y. Extrapyramidal symptoms and advanced calcification of the basal ganglia in a patient with autosomal dominant hypocalcemia. Intern Med. 2013;52:2077-81.

7. Shoback D. Hypoparathyroidism. N Engl J Med. 2008;359:391-403.

8. Betterle C, Garelli S, Presotto F. Diagnosis and classification of autoimmune parathyroid disease. Autoimmun Rev. 2014;13:417-22.

9. Bilezikian JP, Khan A, Potts JT Jr, et al. Hypoparathyroidism in the adult: epidemiology, diagnosis, pathophysiology, target-organ involvement, treatment, and challenges for future research. J Bone Miner Res. 2011;26:2317-37.

10. Sikjaer T, Rolighed L, Hess A, Fuglsang-Frederiksen A, Mosekilde L, Rejnmark L. Effects of PTH(1-84) therapy on muscle function and quality of life in hypoparathyroidism: results from a randomized controlled trial. Osteoporos Int. 2014;25:1717-26.
11. Hadker N, Egan J, Sanders J, Lagast H, Clarke BL. Understanding the burden of illness associated with hypoparathyroidism reported among patients in the PARADOX study. Endocr Pract. 2014;20:671-9.

12. Bollerslev J, Rejnmark L, Marcocci C, et al. European Society of Endocrinology clinical guideline: treatment of chronic hypoparathyroidism in adults. Eur J Endocrinol. 2015;173:G1-120.

13. Benjamin K, Vernon MK, Patrick DL, Perfetto E, Nestler-Parr S, Burke L. Patient-reported outcome and observer-reported outcome assessment in rare disease clinical trials: an ISPOR COA Emerging Good Practices Task Force Report. Value Health. 2017;20:838-55.

14. Coles T, Chen K, Nelson L, et al. Psychometric evaluation of the hypoparathyroidism symptom diary. Patient Relat Outcome Meas. 2019;10:25-36.

15. Hormone Health Network. Hypoparathyroidism. 2018. http://www.hormone.org/questions-and-answ ers/2013/hypoparathyroidism. Accessed 13 Sep 2018. 